\title{
LA CONSTRUCCIÓN DEL "BENEFICIARIO” EN LOS PROGRAMAS SOCIALES DIRIGIDOS A NINTAS, NIÑOS Y ADOLESCENTES EN EL ÁREA METROPOLITANA BONAERENSE
}

\section{THE CONSTRUCTION OF "BENEFICIARY" IN SOCIAL PROGRAMS FOR CHILDREN AND ADOLESCENTS IN METROPOLITAN BUENOS AIRES}

\author{
Valeria Llobet* \\ Cecilia Litichever** \\ Gabriela Magistris***
}

RESUMEN

\begin{abstract}
Este artículo presenta los resultados preliminares de un proyecto de investigación, en el cual se analiza la construcción social de la infancia en los programas sociales ${ }^{1}$. Se busca problematizar la transformación de las políticas sociales, mediante el análisis de programas dirigidos a la infancia y la adolescencia. Las definiciones de problemas, riesgos y destinatarios de los programas, permiten ponderar las formas prácticas de institucionalización del paradigma de derechos humanos.
\end{abstract}

PALABRAS CLAVE: POLÍTICAS SOCIALES * NIÑOS * ADOLESCENTES * EXCLUSIÓN SOCIAL * INCLUSIÓN SOCIAL * DERECHOS DEL NIÑO

\footnotetext{
* Escuela de Humanidades de la Universidad Nacional de San Martín, Argentina. valeria.s.llobet@gmail.com

** Escuela de Humanidades de la Universidad Nacional de San Martín, Argentina. clitichever@flacso.org.ar

*** Becaria de doctorado del Consejo Nacional de Investigaciones Científicas y Técnicas (CONICET), Argentina. gabrielamagistris@hotmail.com
}

$1 \quad$ Proyecto de Investigación Plurianual "La participación de los/as adolescentes en los programas de inclusión social y las identidades de género" financiado por CONICET (Consejo Nacional de Investigaciones Científicas y Técnicas). Directora: Valeria Llobet, Investigadoras: Cecilia Litichever, Florencia Gentile, Marina Medan, Catarina Vilanova, Gabriela Magistris y María Consuelo Gaitán, radicada en el Centro de Estudios sobre Democratización y Derechos Humanos de la Universidad Nacional de San Martín. 


\section{ABSTRACT}

In this article, we present preliminary results of a qualitative research project, which aims to explore the social construction of childhood in social programs. The purpose of this article is to critically approach the transformation of social policy through these programs. We adhere to the general ideational assumptions that the programs' definition of problems, needs and clients express the institutionalization of the human rights discourse in our context. Finally, the central argument is that children's rights discourse is reframed in its institutionalization.

KEYWORDS: SOCIAL POLICY * CHILDREN * ADOLESCENTS * SOCIAL EXCLUSION * SOCIAL INCLUSION * CHILDREN'S RIGHTS

\section{INTRODUCCIÓN}

Los programas de atención a niños, niñas y adolescentes que se desarrollan en áreas sociales gubernamentales en Argentina, siguen actualmente dos grandes líneas rectoras: 1) la protección, cuidado o restitución de los derechos de niños, niñas y adolescentes, 2) la búsqueda de la inclusión o integración social. Ambos ejes confluyen en el mismo período histórico y responden paralelamente a la sanción de la Convención Internacional sobre los Derechos del Niño y a la implementación de políticas de ajuste estructural de la mano con la apelación a los discursos centrados en la pobreza como un problema individual. Con las características propias de cada contexto, la transformación del Estado de Bienestar puede caracterizarse como un proceso mundial. La salida de estas transformaciones en su versión más progresista tiende a vincular el discurso de derechos humanos con el análisis de la pobreza, además de conminar a los estados a integrar la perspectiva de derechos humanos en el diseño de sus políticas sociales ${ }^{2}$ (PNUD, 2000; UNRISID, 2005 y Jorgensen, 2007).

2 Las políticas sociales son definidas por Belmartino et ál., como “(...) un instrumento irremplazable para la producción y conservación del orden, el bienestar y la integración social (...). Podría afirmarse que esa actividad del Estado tiene por finalidad la producción de las condiciones que aseguren la existencia de la sociedad como tal" (2001: 53). En este sentido, participan en la reproducción de la estructura social en tanto "red especial de micropoderes, por medio de la construcción de campos disciplinares, instituciones prestadoras de servicios, un cuerpo de técnicos calificados y un conjunto de prácticas normalizadoras" (Fleury, 1997).
Luego de la crisis, las políticas sociales de atención a los sectores más pobres de la sociedad, parecieron adquirir en el contexto argentino, dos características centrales: si bien, conservan los criterios de focalización en las condiciones de pobreza, extienden la cobertura para tratar de abarcar a casi la totalidad de la población definida como "en situación de exclusión social” (Llobet y Litichever, 2010). Más aún, la inclusión de un programa de ingreso básico, la Asignación Universal por Hijo hacia fines de 2009, implica un avance notable en la dirección de la universalización de ciertas dimensiones de la ciudadanía social, o lo que en el contexto europeo se denominó "universalismo básico" (Lister, 2007). En este sentido, parecería darse cierta similitud con los procesos de ubicación de los "niños" como sujetos prioritarios del bienestar (Watson, 2000) y de las nuevas estrategias de gubernamentalidad (Elizabeth y Larner, 2009).

Por otra parte y paradójicamente, parecería perpetuarse la imposibilidad de salir de los circuitos de asistencia, en especial para la población infantil y adolescente en situación de mayor vulnerabilidad. En este sentido, la mayor preocupación, más que la inclusión social en términos de ciudadanía sustantiva y participación de la producción y ganancias generales de la sociedad, parece ser la inclusión restringida a los programas (Litichever, 2009).

En este contexto, que constituye un desafío para el logro de los derechos de niños, niñas y adolescentes, la hipótesis preliminar es que las políticas sociales se transforman absorbiendo algunos de los nuevos lineamientos sobre el quehacer de la política social. Al mismo tiempo, 
conservan algunos elementos del paradigma ligado al achicamiento del Estado, que se transforman en el acuerdo básico internacional sobre la inviabilidad del "viejo" Estado de Bienestar, incorporan elementos del enfoque de derechos humanos y de un discurso de inclusión social, centrándose -en línea con las transformaciones internacionales- en "el niño" como sujeto del bienestar (Lister, 2006; Dobrowlsky y Lister, 2005; Barrientos y Santibáñez, 2009). En efecto, se enfocan los derechos de niños, niñas y adolescentes como centrales a la hora de (re)definir políticas destinadas a ellos y en general, la protección social. Se considera que ello supone una tensión que se expresará de diferentes modos en algunos aspectos centrales de las definiciones programáticas ${ }^{3}$. Como señaló Jude Fernando, “la ampliación de la brecha entre las expectativas y logros en el área de los derechos humanos señala un asunto fundamental: ¿cuán confortablemente cabe nuestro compromiso con los derechos de los niños/as en nuestras convicciones sobre justicia social?" (2001: 8, traducción propia).

Resumiendo, en este artículo se analiza cómo las definiciones acerca de los beneficiarios y los objetivos de los programas expresan tales tensiones sustantivas a la hora de definir los modelos de interpretación y el alcance que adoptarán los derechos humanos de niños, niñas $\mathrm{y}$ adolescentes.

Se toman para el análisis, los programas de atención a niños, niñas y adolescentes dependientes del Ministerio de Desarrollo Social de la Provincia de Buenos Aires y de la Ciudad de Buenos Aires, Argentina; partidos que concentran casi la mitad de la población menor de 18 años del país. Se trata de un estudio cuya mirada está puesta en discursos, prácticas y relaciones sociales tejidas en el universo simbólico de las políticas de la infancia, contexto que supone tanto una distribución desigual del poder, el cual recrea un orden generacional, de clase y de género, como la institucionalización y legitimación social de esta distribución. La especificidad

$3 \quad$ Se entiende por definiciones programáticas todos aquellos aspectos expresados en documentos institucionales donde se hace referencia a los objetivos, metodología de trabajo, población con la que trabajan cada uno de los programas, etc. de este estudio, respecto al campo de estudios sobre el tema de políticas de infancia notablemente desarrollado, es tanto de enfoque teórico (y correlativamente de objeto) como de nivel de análisis (si bien, incluye el nivel micro-social, aspira a integrarlo en un nivel meso-social o de "campo") ${ }^{4}$.

El tipo de diseño es "instrumental de casos múltiples"5 (Yin, 1993), en tanto se procura comprender un problema general, la construcción social de la infancia en las políticas sociales, a partir del estudio de unos casos particulares (Stake, 1998), las políticas y programas con explícitos objetivos de inclusión social. Articula con una perspectiva de etnografía ampliada (Haney, 2002), estrategias de observación participante en contextos de implementación, entrevistas en profundidad y grupales a los agentes y los beneficiarios, así como análisis documental. Ello dado que se considera importante recuperar el carácter de múltiples perspectivas, propio de los estudios de caso (Tellis, 1997). Aquí se presenta un análisis exploratorio centrado solamente en las definiciones programáticas de los programas presentes en la documentación oficial, así como, entrevistas a informantes clave. La estrategia analítica se basa en el análisis

4 Es necesario resaltar que el campo de las políticas en las que se construyen los "casos" implica los procesos de conformación y estabilización de esquemas interpretativos y marcos de significación que orientan las acciones y las decisiones que se analizan, así como, las disputas entre actores por tales interpretaciones (Bourdieu y Wacquant, 2005; Fraser, 1991).

5 El caso cumple el rol de mediación para la comprensión de un fenómeno que lo trasciende y es un instrumento para evidenciar las características del mismo. En este sentido, el conjunto de casos - programas- permite, a partir del análisis de sus particularidades $y$ de las relaciones entre sus propiedades, desarrollar algunas hipótesis interpretativas o "generalizaciones analíticas" (Yin, 1993), respecto a los procesos de construcción social de la infancia y la adolescencia en el campo de las políticas para la infancia. En este sentido, si bien se reconocen las limitaciones metodológicas de los estudios de caso, se las intenta sortear mediante la consideración de heterogeneidades teóricamente significativas, tales como, las distintas definiciones de población objetivo y la selección de casos múltiples. 
pragmático-crítico del discurso (Fraser, 1991), que permitirá integrar los distintos niveles y perspectivas $^{6}$.

\section{LAS POLÍTICAS SOCIALES PARA LA NIÑEZ Y LA ADOLESCENCIA. EL ENFOQUE DE DERECHOS}

La dimensión simbólica implícita en las políticas da cuenta de la forma en que la intervención del Estado opera desde una determinada interpretación de las necesidades, en el marco de un discurso legítimo construido desde los discursos expertos institucionalizados en el Estado (Fraser, 1991 y Haney, 2002). Las definiciones sobre problemas, poblaciones $y$ objetivos programáticos son tratadas como cristalizaciones de las operaciones del poder y como prácticas sociales institucionalizadas (Foucault, 1978; Fraser, 1991 y Padamsee, 2009). De este modo, las definiciones analizadas conllevan una potencia prescriptiva que delimita las acciones posibles, las soluciones imaginadas, el sentido común institucional y expresa modalidades dominantes en que se conciben los problemas (Llobet $y$ Litichever, 2010).

Las políticas de atención a la infancia vigentes a partir de la adopción de la Convención Internacional sobre los Derechos del Niño, tienen el compromiso de respetar y garantizar los derechos de la infancia (García, 1997 y Baratta, 1999), así como, el Estado debe proteger y velar por el cumplimiento de los derechos

$6 \quad$ De acuerdo con Fraser (1991), es posible rastrear en los discursos sobre necesidades, sujetos y cursos de acción de la política pública, un conjunto de operaciones prácticas en las que se definen las disputas sobre la significación. De este modo, el análisis de las unidades discursivas no es solo intrínseco a sí mismo —exclusivamente textual—, sino que se le inscribe en las redes de relaciones en las cuales es producido, incluyendo las instituciones sociales que dan cuenta. En tanto, todo discurso es un discurso que legitima o deslegitima las instituciones sociales. Los procedimientos suponen identificar las unidades de sentido que construyen las definiciones sobre los núcleos temáticos: necesidades, destinatarios, prestaciones, riesgo, etc. además de establecer las redes de significación de los programas sobre las mismas, ponderándolas críticamente para hacer emerger contradicciones y tensiones. de los niños (Cillero, 2001). No obstante, en otras investigaciones se ha notado que el discurso de derechos, en determinados contextos, puede promover la despolitización de las problemáticas sociales adolescentes y desvincularlas de su dimensión económica así como de la dimensión de la desigualdad ${ }^{7}$ (Llobet, 2010).

Una tendencia investigativa local ha sido ubicar los problemas del enfoque de derechos de la infancia, en la falta de modernización del Estado y sus agentes, como señala críticamente Villalta (2006) o en las motivaciones de control social (Guemureman y Daroqui, 2001). Desde nuestra perspectiva, por el contrario, es en el plano de las definiciones prácticas donde debe analizarse tal eficacia. Como señalan Fonseca y Cardarello (2005), el análisis del enfoque de derechos en su eficacia práctica es también el análisis del conjunto de operaciones epistemológicas y discursivas que lo despliegan.

Resumiendo, revisar los ejes de las "políticas de infancia" (Therborn, 1996), es decir, el conjunto de definiciones políticas institucionalizadas respecto a lo que será definido como "niño", los derechos que le son atribuidos y las necesidades que son legitimadas institucionalmente, es de relevancia para comprender los sujetos que están siendo construidos en estas, así como, las redefiniciones de sus derechos.

\subsection{PLANES Y PROGRAMAS EN EL ÁREA METROPOLITANA}

El área metropolitana (Ciudad Autónoma de Buenos Aires y partidos del conurbano bonaerense) es señalada como el área de mayor concentración de población menor de 18 años en situación de pobreza del país. Los datos disponibles en fuentes oficiales indican que al año 2006, el 39, 4\% de los niños(as) menores de 14 años que vivían en el área metropolitana (ciudad de Buenos Aires y provincia de Buenos Aires) se encontraban en situación de pobreza (Instituto Nacional de Estadística y Censos-INDEC, de acuerdo a los datos de la Encuesta Permanente de Hogares-

\footnotetext{
$7 \quad$ Entendida como la desigual distribución y apropiación de las posibilidades de acceder a bienes culturales, materiales y oportunidades.
} 
EPH Continua Segundo Semestre de 2006). A su vez, según UNICEF (United Nations Children's Fund), al año 2011, el 17, 3\% de los niños $y$ adolescentes en Argentina, vivían en condición de pobreza.

La Ciudad Autónoma de Buenos Aires (en adelante CABA), concentra la atención a la niñez y la adolescencia a través de las acciones del
Ministerio de Desarrollo Social y del Consejo de Derechos de Niños, Niñas y Adolescentes ${ }^{8}$. El primero, en su Dirección General de Niñez y Adolescencia ${ }^{9}$, articula cuatro líneas de acción: Atención en Situación de Vulnerabilidad Social, Desarrollo Integral, Programa Adolescencia y Fortalecimiento de Vínculos, de las cuales se desprenden 16 programas.

CUADRO 1

LÍNEAS DE ACCIÓN Y OBJETIVOS DE LA CABA

\begin{tabular}{lll}
\hline LINEA DE ACCIÓN & MISION Y OBJETIVOS & PROGRAMAS \\
\hline Atención a La Niñez & Implementar circuitos y programas de atención & Equipo móvil \\
y Adolescencia & directa, tanto en la calle como en centros & Centro de Atención Integral a la Niñez y \\
en Situación de & institucionales para todos los niños, niñas y & Adolescencia (CAINA) \\
Vulnerabilidad Social & adolescentes en situación de vulnerabilidad & Paradores \\
& social en la ciudad de Buenos Aires, a fin de & Hogares propios \\
& garantizar el ejercicio de sus derechos y la & Hogares conveniados \\
& restitución de aquellos que les hayan sido &
\end{tabular}
vulnerados.

\begin{tabular}{|c|c|c|}
\hline Desarrollo Integral & $\begin{array}{l}\text { Promover el desarrollo integral de niños, niñas y } \\
\text { adolescentes a través de su inclusión en políticas } \\
\text { públicas básicas como educación, juego y } \\
\text { recreación: } \\
\diamond \quad \text { acompañamiento a las familias en la crianza } \\
\diamond \quad \text { fortalecimiento de la red vincular de niños } \\
\text { y adolescentes en los barrios y zonas menos } \\
\text { favorecidas } \\
\diamond \quad \text { promoción del aprendizaje y creación de un } \\
\quad \text { vínculo para el seguimiento de la inclusión }\end{array}$ & $\begin{array}{l}\text { Centros de Desarrollo Integral (CeDIs) } \\
(21) \\
\text { Centros de Acción Familiar (CAF) (8) } \\
\text { Casas de Niños, Niñas y Adolescentes (7) } \\
\text { Actividades recreativas de integración } \\
\text { social (Juegotecas) (18) } \\
\text { Puerto Pibes }\end{array}$ \\
\hline Programa Adolescencia & & Transferencias Monetarias \\
\hline $\begin{array}{l}\text { Fortalecimiento de } \\
\text { Vínculos }\end{array}$ & $\begin{array}{l}\text { Prevenir y dar respuestas efectivas a las } \\
\text { situaciones de vulneración de derechos de niños, } \\
\text { niñas y adolescentes en el ámbito de la Ciudad } \\
\text { de Buenos Aires. } \\
\text { El trabajo se centra en las causas que generan } \\
\text { exclusión y situaciones de riesgo dentro del } \\
\text { ámbito familiar y comunitario, apuntando } \\
\text { a potenciar la posibilidad de cambio en las } \\
\text { personas. }\end{array}$ & $\begin{array}{l}\text { Equipos zonales de fortalecimiento y } \\
\text { revinculación. } \\
\text { Inclusión socioeducativa y laboral } \\
\text { (Cooperativa de Producción y } \\
\text { Aprendizaje-COOPA, Bajo Flores y Lazos) } \\
\text { Reconstruyendo Lazos } \\
\text { Acompañantes hospitalarios y } \\
\text { terapéuticos. } \\
\text { Explotación sexual comercial }\end{array}$ \\
\hline
\end{tabular}

Fuente: Elaboración propia con base en los datos del Ministerio de Desarrollo Social CABA.

$8 \quad$ Este último es un organismo descentralizado creado por la Ley 114, cuya función es verificar y transversalizar el cumplimiento del enfoque de derechos de la infancia en el conjunto de las acciones estatales. Cuenta con oficinas de recepción de reclamos por vulneración de derechos de los niños(as), además con Defensorías de Niños, Niñas y Adolescentes, $y$ desarrolla una mesa intersectorial, denominada Plenario, en la que participan los distintos ministerios de gobierno, legisladores de la comisión de infancia y organizaciones de la sociedad civil.
$9 \quad$ Las líneas de acción y programas han sufrido notables transformaciones en los últimos años, en parte, debido al cambio de estatus de la jurisdicción, que pasó de ser un distrito federal a ser un distrito autónomo. Las acciones que tienden a ubicarse como "fundacionales" encuentran al Centro de Atención Integral a la Niñez y Adolescencia (CAINA) como la única instancia que persiste desde los lejanos inicios de la década de 1990 y los debates iniciáticos respecto a los derechos del niño en el ámbito local. 
La estructuración de la asistencia brindada parece vincularse con supuestos de gradación de la vulnerabilidad social y con estrategias generales de intervención. De este modo, aún cuando sus enunciados generales sobre la misión y objetivos apelan a un lenguaje de derechos, este no aparece cuestionando la institucionalidad. En efecto, una manera de interpretar la eficacia de la institucionalización del enfoque, es su capacidad para reconfigurar la propia formulación de áreas de acción en el organigrama. Si bien, esta reconfiguración programática es redefinida en las negociaciones entre actores (Villalta, 2010), el peso de la institucionalidad es fuerte a la hora de limitar las posibilidades de acción de los agentes, incluso en el plano de sus posibilidades de interpretación e intervención (Llobet, 2007).

Por otra parte, desde 2005, la CABA desarrolla el programa de Transferencia Condicionada de Ingresos (TCI) "Ciudadanía Porteña" —creado por ley en el año 2005 y aún vigentedirigido a "disminuir los niveles de desigualdad en la Ciudad Autónoma de Buenos Aires (...) mediante un subsidio mensual que mejora el ingreso de los hogares en situación de pobreza o indigencia”. Si bien, el programa privilegia a las mujeres argentinas ${ }^{10}$ como titulares del

10 De acuerdo a la información oficial del programa, "los hogares cuyos miembros mayores de 18 años al momento de la inscripción carecían de documentación argentina realizaron una preinscripción al Programa; el trámite de inscripción se completa cuando regularizan su situación migratoria; lo mismo sucede con el resto de integrantes del hogar, que para poder ser incluidos en la solicitud deberán haber iniciado la tramitación del documento argentino que acredite identidad". beneficio, la carta compromiso firmada por las mismas da cuenta de un foco en infancia y adolescencia ${ }^{11}$, como la mayoría de los programas de TCI. De este modo, de acuerdo con las evaluaciones del mismo, cubría a diciembre de 2009 a 70 mil hogares, los cuales abarcaban $260 \mathrm{mil}$ personas, de las cuales el 22\% tenía entre 15 y 25 años, en tanto que casi la mitad del total de las personas beneficiarias son menores de 18 años, alcanzando según las estimaciones, a casi la totalidad de personas menores de 18 años en situación de pobreza por ingresos/habitantes de la CABA (UIMyE, MDS, 2010).

Por su parte, la Provincia de Buenos Aires desarrolla acciones para la población menor de 18 años desde la Subsecretaría de Políticas Sociales ${ }^{12}$ y desde la Subsecretaría de Niñez y Adolescencia.

$11 \quad$ La misma impone las siguientes obligaciones: realizar los controles de salud de embarazadas, niños $y$ adolescentes del hogar. Garantizar la asistencia escolar de todos los niños y adolescentes de 5 a 18 años (inclusive) del hogar, sin excepciones. Brindar información verdadera sobre la composición y condiciones del hogar y de sus miembros, así como de las modificaciones que puedan surgir: fallecimientos, salida de uno de los miembros de la unidad de convivencia, miembros presos, nacimientos, etc. No omitir información ni falsificar documentos o certificados que solicite el Programa. Hacer una adecuada utilización de los recursos del Programa. Cumplir las normas sobre prohibición del trabajo infantil en sus diversas formas. Asistir a las reuniones convocadas por el Programa.

12 La primera en la provincia en generar acciones de este tipo, a partir de la institucionalización y reorganización del iniciático "Plan Vida", creado por Hilda González de Duhalde durante la gobernación de su esposo en la década de 1990. 


\section{CUADRO 2 \\ LÍNEAS DE ACCIÓN Y OBJETIVOS \\ PROVINCIA DE BUENOS AIRES}

\begin{tabular}{|c|c|c|}
\hline $\begin{array}{l}\text { SUBSECRETARÍA O } \\
\text { SISTEMA }\end{array}$ & MISION Y OBJETIVOS & PROGRAMAS \\
\hline \multirow[t]{3}{*}{$\begin{array}{l}\text { Subsecretaría de } \\
\text { Políticas Sociales }\end{array}$} & $\begin{array}{l}\text { Mejorar las condiciones de nutrición, } \\
\text { crecimiento y desarrollo de la población } \\
\text { materno-infantil, fortaleciendo } \\
\text { las capacidades de las familias y } \\
\text { consolidando redes sociales. }\end{array}$ & Plan Más Vida \\
\hline & $\begin{array}{l}\diamond \quad \text { Brindar atención alimentaria, } \\
\text { estimulación y apoyo al proceso } \\
\text { enseñanza-aprendizaje de niños de } 0 \text { a } \\
14 \text { años. }\end{array}$ & Unidades de Desarrollo Infantil \\
\hline & $\begin{array}{l}\diamond \text { Implementar la CDN y el enfoque de } \\
\text { género. }\end{array}$ & $\begin{array}{l}\text { Programa Crecer Jugando en Igualdad de } \\
\text { Oportunidades }\end{array}$ \\
\hline \multicolumn{3}{|c|}{ SUBSECRETARÍA DE NIÑEZ Y ADOLESCENCIA } \\
\hline $\begin{array}{l}\text { Sistema de Promoción y } \\
\text { Protección de Derechos }\end{array}$ & $\begin{array}{l}\diamond \quad \text { Promover la desconcentración de las } \\
\text { acciones de promoción, protección } \\
\text { y restablecimiento de derechos en el } \\
\text { ámbito municipal, con participación } \\
\text { activa de las organizaciones no } \\
\text { gubernamentales de atención a la niñez. }\end{array}$ & $\begin{array}{l}\text { 1.a Servicio Local de Promoción y } \\
\text { Protección de Derechos (SLPPD) } \\
\text { 1.b Servicios Zonales Promoción y } \\
\text { Protección de Derechos (SZPPD) } \\
\text { 2. Programa de Abordaje Múltiple para } \\
\text { Niños, Niñas y Adolescentes (PAM), La Plata } \\
\text { 3. Servicio de Atención Terapéutica Integral } \\
\text { (SATI), La Plata } \\
\text { 4. Centro de Tratamiento y Atención } \\
\text { Integral (CTAI), La Plata } \\
\text { 5. Servicio Educativo de Apoyo Escolar } \\
\text { Externo "Puertas Abiertas", La Plata } \\
\text { 6. Servicio de Asistencia Familiar (SAF), La } \\
\text { Plata } \\
\text { 7. Centro Terapéutico Diurno (CTD), La Plata } \\
\text { 8. Programa de Autovalimiento en la } \\
\text { Crianza. Hogar Evita - Centro de Día- La } \\
\text { Plata } \\
\text { 9. Programa de Autovalimiento Juvenil } \\
\text { (PROA), La Plata y Lomas de Zamora } \\
\text { 10. Programa "Barrio Adentro", Altos de San } \\
\text { Lorenzo, La Plata } \\
\text { 11. Programa de Becas Niñez }\end{array}$ \\
\hline $\begin{array}{l}\text { Sistema de } \\
\text { Responsabilidad Penal } \\
\text { Juvenil }\end{array}$ & $\begin{array}{l}\text { Formular, coordinar y ejecutar } \\
\text { políticas, programas y medidas } \\
\text { destinadas a prevenir el delito juvenil, } \\
\text { asegurando los derechos y garantías de } \\
\text { los jóvenes infractores a la ley penal, } \\
\text { además de generar ámbitos para la } \\
\text { ejecución de medidas socioeducativas } \\
\text { que, centradas en la responsabilidad } \\
\text { del joven infractor, posibiliten su real } \\
\text { inserción en su comunidad de origen. }\end{array}$ & $\begin{array}{l}\text { 1. Centros de Recepción } \\
\text { 2. Centros Cerrados } \\
\text { 3. Centros de Contención } \\
\text { 4. Centros de Referencia }\end{array}$ \\
\hline $\begin{array}{l}\text { Programa de } \\
\text { Responsabilidad Social } \\
\text { Compartida Envión }\end{array}$ & $\begin{array}{l}\diamond \quad \text { "Integrar a estos chicos al sistema } \\
\text { educativo y enseñarles un oficio, además } \\
\text { de procurarles un espacio de afecto } \\
\text { y contención donde puedan realizar } \\
\text { actividades deportivas, recreativas y } \\
\text { culturales con la guía de profesionales } \\
\text { idóneos". }\end{array}$ & $\begin{array}{l}\text { 1. Envión Educativo } \\
\text { 2. Envión de Vida } \\
\text { 3. Asociación Civil Envión }\end{array}$ \\
\hline
\end{tabular}

Fuente: Elaboración propia con base en los datos del Ministerio de Desarrollo Social, Provincia de Buenos Aires. 
La nueva estructura de la Secretaría de Niñez da cuenta de su adecuación funcional a los preceptos de la Ley Nacional de Protección Integral y a su incorporación en la Ley Provincial. De acuerdo a la información oficial ${ }^{13}$, el Sistema de Protección Integral atiende anualmente a unos 50000 niños, niñas y adolescentes menores de 18 años; el Sistema de Responsabilidad Penal a $2760^{14}$ y el Programa de Responsabilidad Social Compartida Envión contaba a mediados de 2010 , con casi 60 sedes $^{15}$. No obs-

13 Véase la página del Ministerio de Desarrollo Social http://www.desarrollosocial.gba.gov.ar/programas/ index.php.

14 Según las estimaciones del propio Ministerio, el Sistema de Protección destina 190 millones de pesos anuales, es decir, un total anual de $\$ 3800$ (casi U\$1000) por niño, niña o adolescente. En tanto, el Sistema de Responsabilidad Penal destina alrededor de 110 millones de pesos anuales para la asistencia de 2760 adolescentes, es decir, un total de casi $\$ 40000$ (U\$10 000) anuales per capita.

15 Es difícil, no obstante, conocer la cantidad de beneficiarios(as). En la página web del Ministerio se informa la intención de alcanzar los 50000 destinatarios durante 2010, pero entrevistas a informantes clave indicaban que la cobertura no superaba los 5000 adolescentes, lo cual se condice tante, interesa señalar la persistencia de una línea de interpretación más vinculada a los enfoques de necesidades que al enfoque de derechos, que emerge de la distribución de programas por secretarías. En efecto, una de ellas conserva (aunque remozada) la perspectiva de desarrollo psico-biológico y moral que inspiró los primeros programas, y la otra despliega unas formulaciones más acordes a la legislación actual ${ }^{16}$.

De este modo, es necesario revisar de qué manera las dimensiones estructurantes de los programas configuran campos semánticos respecto a la infancia y la adolescencia. De la totalidad de programas, se seleccionan los siguientes cuatro, cuya población se encuentra entre las que mayores vulneraciones de derechos soportan, o como en el caso del programa Envión, se trata de uno de los programas que aspira a adquirir mayor cobertura a nivel de la provincia de Buenos Aires. Se detallan algunas descripciones sobre la población destinataria y los objetivos, tal como se reflejan en el cuadro 3.

más con la cantidad de sedes, de tutores (301) y de operadores en equipos multidisciplinarios (402). necesariamente excluya estas perspectivas. 
CUADRO 3

POBLACIÓN DESTINATARIA Y OBJETIVOS DE LOS PROGRAMAS

\section{NOMBRE DEL PROGRAMA DEPENDENCIA INSTITUCIONAL}

Programa Envión Subsecretaría de Niñez y Adolescencia. Ministerio de Desarrollo Social Provincia de Buenos Aires, Argentina.

\author{
POBLACIÓN \\ DESTINATARIA
}

Adolescentes y jóvenes entre 12 a 21 años en situación de vulnerabilidad social.
OBJETIVOS

Garantizar el acceso a recursos y oportunidades para el desarrollo personal y la integración social de los niños, adolescentes y jóvenes. El objetivo es promover la inclusión social de los chicos a través del desarrollo de sus capacidades, la adquisición de nuevos saberes y habilidades. La incorporación de nuevas modalidades de relación y vinculación con el medio social, ampliando la apertura de posibilidades para que puedan elegir y desarrollar un proyecto de vida que supere los condicionamientos sociofamiliares de origen.

Ofrecer a niñas, niños y adolescentes que viven en la calle un espacio institucional de referencia $y$ atención desde la Protección Integral, a partir del cual puedan elaborar desde sus singularidades y en forma conjunta, diferentes estrategias que apunten a mejorar la calidad de vida, a disminuir los riesgos y vulneración de derechos de la situación de calle y a un alejamiento paulatino de la misma.

de Desarrollo Social Ciudad Autónoma de Buenos Aires.

\section{Niños(as) en situación} de calle

Subsecretaría de Niñez $y$ Adolescencia.

Ministerio de Desarrollo

Social Provincia de Buenos Aires.
Niñas, niños y adolescentes en situación de calle en ciudad de Buenos Aires, cuyas edades oscilan entre los 8 y 18 años.

Niños(as) de 0-18 años en situación de calle y/o alta vulnerabilidad psicosocial con o sin causa judicial.

\section{Objetivo General}

$\diamond \quad$ Prevenir los factores de riesgo que conlleven a los niños y adolescentes a la situación de calle.

$\diamond$ Reducir los efectos de los factores de riesgo a los que se encuentran expuestos los niños y adolescentes en situación de calle, desde una perspectiva de atención integral, revalorizando el lugar de la familia y su comunidad. Objetivos Específicos

$\diamond$ Mejorar las condiciones de contención afectiva, salud, educación, posibilidades de capacitación y acceso al mercado laboral e integración familiar de los niños y adolescentes en situación de calle y/o en situación de alta vulnerabilidad psicosocial, comprendidos en las áreas de influencia del Programa.

$\diamond \quad$ Prevenir la expulsión del ámbito familiar y comunitario de niños que viven en distritos con indicadores socioeconómicos críticos, evitando su ingreso al circuito de la calle.

$\diamond$ Desarrollar estrategias de sensibilización y difusión de la problemática tendiente a instalar en el colectivo social una posición actitudinal de mayor compromiso.

$\diamond$ Optimizar y articular recursos existentes en el ámbito departamental, Municipal y comunitario, superando respuestas atomizadas, parcializadas y superpuestas en la atención al chico en situación de calle y/o alta vulnerabilidad psicosocial.

$\diamond \quad$ Prevenir la utilización de niños y adolescentes en situación de calle, por parte de adultos y organizaciones que lucren con la mendicidad, el trabajo y/o la explotación sexual de los niños. 


\begin{tabular}{|c|c|c|}
\hline $\begin{array}{l}\text { NOMBRE DEL PROGRAMA } \\
\text { DEPENDENCIA } \\
\text { INSTITUCIONAL }\end{array}$ & $\begin{array}{c}\text { POBLACIÓN } \\
\text { DESTINATARIA }\end{array}$ & OBJETIVOS \\
\hline Paradores & $\begin{array}{l}\text { Niños, niñas y adolescentes } \\
\text { de y en la calle hasta los } 18 \\
\text { años de edad. }\end{array}$ & $\begin{array}{l}\diamond \quad \text { Atender las necesidades inmediatas de alimento y/o } \\
\text { descanso. } \\
\diamond \quad \text { Brindar un ámbito seguro que resguarde la integridad } \\
\text { física, moral y emocional. } \\
\diamond \quad \text { Adoptar las medidas necesarias tendientes a la } \\
\text { contención del niño, niña o adolescente en su núcleo } \\
\text { familiar o bien la familia ampliada, facilitando alternativas } \\
\text { para su revinculación. } \\
\diamond \quad \text { Detectar si son objeto de violencia, maltrato y/o } \\
\text { explotación y en caso de ser necesario, ponerlos en } \\
\text { contacto con los organismos pertinentes. } \\
\diamond \quad \text { Brindar información y asesoramiento sobre sus } \\
\text { derechos. } \\
\diamond \quad \text { Propiciar procesos de mejora de la autoestima. } \\
\diamond \quad \text { Propender al desarrollo de sentimientos de pertenencia } \\
\text { a colectivos y estimular la conciencia crítica. } \\
\diamond \quad \text { Promover la creatividad y la capacidad de realización. }\end{array}$ \\
\hline
\end{tabular}

Fuente: Elaboración propia a partir de datos oficiales.

El Programa Envión ${ }^{17}$ aparece como una instancia de transición interesante, porque de su origen municipal y su carácter de "programa bandera" de la actual gestión de desarrollo social provincial ${ }^{18}$, pasó a ser cofinanciado por el Consejo Federal de Niñez y Adolescencia mediante una Addenda firmada por el gobierno provincial. De este modo, se permite su expansión y se garantiza una instancia de monitoreo. El CAINA ${ }^{19}$, a su vez, ha

17 El mismo reemplazó desde el año 2009, al Proyecto Adolescente, el cual se encontraba en curso desde 2007, a su vez este resultó una modificación y extensión del Proyecto Comunidades Vulnerables. En todos los casos se trataba de programas de transferencias condicionadas de ingresos, cuya condicionalidad se vinculaba con la asistencia a talleres, grupos de jóvenes, programas de formación informal, etc.

18 El problema de los jóvenes de sectores populares comenzó con la gestión del ex ministro de Desarrollo Social bonaerense, Daniel Arroyo, quien para fundamentar la inversión del programa anterior, el Proyecto Adolescente, planteaba a los medios que vivían (en 2007 y 2008) en la provincia de Buenos Aires, 500000 jóvenes de los cuales más de 100000 se encontraban en "situación de exclusión institucional".

19 El CAINA fue creado en el año 1992, como uno de los primeros programas luego de la ratificación de sido el programa iniciático de la ciudad de Buenos Aires, con casi 20 años de desarrollo, período en el cual han variado notablemente tanto sus objetivos como su misión. Los paradores y los centros para niños en situación de calle de la provincia son dos instancias que llevan también varios años de implementación.

En base a estos programas y a partir de hallazgos anteriores, respecto a la manera en que los programas sociales construyen las necesidades e identidades infantiles, se presentaran algunos ejes de definición de tales cláusulas para comprender la construcción de los "beneficiarios"; es decir, del conglomerado de significaciones disponibles para definir "la infancia", los distintos programas enfatizan algunos ejes en determinados momentos, señalando lo que va a conformar sus operaciones clasificatorias (Llobet, 2006). Se trata de la puesta en juego de criterios relativos a las condiciones de vida, a definiciones morales y a las estrategias a desarrollar.

la CDN en la Argentina, el cual sigue funcionando en la actualidad. 


\section{SOBRE LOS PROBLEMAS Y LOS SUJETOS, O CÓMO DEFINEN LOS PROGRAMAS A LA POBLACIÓN QUE DICEN ATENDER}

Los criterios de selección de la población con la que se trabaja, tienen que ver con las condiciones sociales de vida de la misma, las cuales son definidas por los programas de vulnerabilidad (social o psicológica), exclusión o riesgo. Los programas de atención a la infancia analizados parecen considerar como condiciones dadas, a algunas tales como "vulnerabilidad" y "riesgo", sin explicitar qué se entiende por cada uno de ellos: ¿qué significa "vulnerabilidad social"? En otras investigaciones se han hallado que las significaciones prácticas que estos "criterios" adquieren, no solo son variables, sino que las interpretaciones de los agentes suelen vincularlas con criterios morales de definición de la normalidad, antes que con criterios sociales de valoración de la desigualdad, la cual se ha denominado la psicologización de las políticas para la infancia (Llobet, 2006 y 2009).

A su vez, los sujetos realizan adaptaciones para poder ser parte del grupo social que las políticas dicen atender. Estas adaptaciones estarían orientadas a demostrar que son sujetos atendibles por las mismas. En este sentido, en otras investigaciones de integrantes del equipo de investigación (Llobet, 2009a; Gentile, 2007 y Litichever, 2009) se ha hallado que la población definida como "chicos en situación de calle", adquiere diversos significados de acuerdo a los actores y las instituciones. Por un lado, la "situación de calle" es dinámica, en tanto los niños y adolescentes pasan períodos en sus casas y otros en la calle. No obstante este dinamismo, se dan procesos de identificación con los requerimientos institucionales de permanencia. Así, muchas veces los requisitos para ser "beneficiarios" solo en tanto "chicos de la calle", puede llevar a diferentes negociaciones identitarias, algunas de ellas altamente conflictivas (Litichever, 2009 y Llobet, 2006).

Asimismo, los programas definen como estrategias para la inclusión social y/o la restitución de derechos, entre otras herramientas, el desarrollo de las capacidades, la autovaloración y un proyecto de vida alternativo a los condicionamientos que brinda la familia de origen.

La adolescencia es considerada, asî como un momento del proceso de consolidación de un proyecto de vida. Es por ello que los objetivos centrales de la mayoría de los programas sociales dirigidos a ellos, se basan en el acompañamiento a las y los adolescentes para tal establecimiento de un proyecto vital-profesional, como paso previo a la inclusión social efectiva, en tanto expresión singular de una demanda social (Llobet, 2009). Llama la atención en este punto, cómo los cambios requeridos para la inclusión social de los sectores postergados, parecen estar en manos de la propia población destinataria. En ningún momento se hace mención a las características de las instituciones (a excepción de la familiar), teniendo algún papel en aquello que es denominado genéricamente como exclusión.

Ahora bien, si los programas de atención a la infancia consideran al niño, niña o adolescente como un sujeto aislado y vulnerado/vulnerable, aunque probablemente puedan desarrollar acciones tendientes a la protección o restitución de derechos, difícilmente logren los objetivos de inclusión social. La paradoja que se presenta es que por más que estos objetivos no sean cubiertos, sí es posible para los programas garantizar el orden y la reproducción de la sociedad. En este sentido, parecería corroborarse el planteo de que las nuevas políticas sociales colocan en el centro una figura de "niño" que reorganizaría las estrategias de gubernamentalidad (Elizabeth y Larner, 2009).

De este modo, se observa como se construye en los programas sociales analizados, una definición abstracta de los/as beneficiarios/as, en tanto la definición empírica de quien sería un adolescente en "situación de vulnerabilidad social" depende de una definición de la vulnerabilidad. Ello dado que la vulnerabilidad social a la que se apela en los diseños programáticos, es la vulnerabilidad de las y los adolescentes a sus condiciones materiales y sociales de existencia. Condiciones que no procuran ser problematizadas por los programas en sus acciones, más allá de que se integren mediante una semántica crítica en sus fundamentos y orientaciones. 


\subsection{SOBRE LOS OBJETIVOS}

Los objetivos de los programas redundan en la identificación de necesidades de contención, prevención del riesgo, pertenencia a un colectivo, mejorar la autoestima, incorporación de nuevas modalidades de relación, etc. De este modo, el discurso experto (Fraser, 1991), establece que estas son las necesidades de la "población vulnerable". La exclusión social parece responder a carencias personales (privadas y despolitizadas) y la inclusión social parecería estar garantizada por ciertas modificaciones actitudinales de los sujetos.

En este sentido, los objetivos de los programas van desde la contención a la adquisición de nuevos saberes y capacidades que garantizarían la inclusión social. La contención comprende servicios concretos como baño $y$ comida o intervenciones frente a situaciones de maltrato o abuso. La adquisición de nuevos saberes y capacidades no aparece especificada en los objetivos de los programas (excepto en la reformulación del Programa Envión), se trata más bien de generalidades relacionadas con los cambios personales de los sujetos. En los casos en que aparecen mencionados tales nuevos saberes, se atribuye a los mismos la capacidad de generar tanto los conocimientos como las actitudes "necesarias para insertarse laboralmente" (Programa Envión Educativo), convergiendo en señalar que la inclusión social es un problema de actitudes.

A pesar de las características similares que comparten los programas analizados, es posible visualizar algunas diferencias entre las dos jurisdicciones seleccionadas. Mientras que la Ciudad de Buenos Aires no hace referencia en sus objetivos, ni a la comunidad en términos de inclusión ni a los propios programas en términos de proveer servicios de manera articulada, no obstante en algunos casos, la Provincia de Buenos Aires hace mención directa a estos aspectos. El trabajo con la comunidad se explicita en términos de prevención y sensibilización para evitar situaciones de explotación y buscar mayor "compromiso" de la sociedad. A su vez, se menciona directamente la necesidad de que los programas funcionen articuladamente para garantizar determinados recursos. Se puede concluir, provisoriamente, que existe en este distrito una mayor predisposición, al menos prescriptivamente, para trabajar con la comunidad $y$ para establecer mecanismos de articulación entre los programas.

De todos modos, es importante aclarar que la apelación a la comunidad aparece en dos direcciones: por un lado, desde la prevención y concientización de diversas problemáticas sociales que afectan a niños, niñas y adolescentes y por otro, como lugares sobre los cuales incidir, en tanto forman parte del entorno $y$ es allí donde se encuentran indicadores de pobreza que afectan al cumplimiento de los derechos de niños, niñas y adolescentes.

En este sentido, se trae aquí la existencia de distintas formas de considerar al territorio/ comunidad donde se desenvuelven los jóvenes. Así, se puede visualizar al territorio como fuente de desigualdades locales y segregación territorial (Saraví, 2006) o bien, partir de entender que la inscripción territorial es un rasgo distintivo de las nuevas clases populares $y$ por tanto, de su modo específico de integración y afiliación, transformándose en la base principal de la estabilización de la experiencia (Merklen, 2005).

Contrastan en este sentido, los dos programas de atención a chicos y chicas en situación de calle. Mientras que el programa correspondiente a la ciudad de Buenos Aires se refiere centralmente a la atención individual de los niños, niñas y adolescentes que van a la institución; en la provincia de Buenos Aires, se hace mención a las familias y comunidades con indicadores socioeconómicos críticos, sobre las cuales el programa debería intervenir para evitar que niños, niñas $y$ adolescentes ingresen al circuito de la calle. La apelación a la comunidad, no obstante, no resulta clara. Puede ser pensada como equivalente social de "territorio", en el sentido de que permite visualizar geográfica y socialmente la necesidad de intervención. En este sentido, algunas referencias de las directrices nacionales (Ministerio de Desarrollo Social, 2007) en el ámbito de la provincia de Buenos Aires, podrían permitir pensar que para el Ministerio de Desarrollo Social, la "comunidad" es un actor geográficamente situado al que hay que fortalecer para que funcione como "contenedor" 
de las familias. Por supuesto, ese "actor" como tal no existe, se trata de un conjunto heterogéneo $y$ conflictivo de actores sociales que, por más que tengan existencia en un territorio, no necesariamente lo componen como comunidad. Es necesario profundizar en este aspecto, en tanto puede estar significando más bien una forma equivalente a la "ongeización" de las políticas para la infancia, en clave barrial.

Por otra parte, la apelación a la intervención en comunidades con indicadores socioeconómicos críticos puede entenderse tanto como un dato "dado" y por lo tanto naturalizado, o con el objetivo de transformar esas situaciones críticas por las que atraviesan los niños, niñas y adolescentes.

En otro orden, la familia aparece mencionada en los objetivos de los programas de diversas maneras. En general, se le considera como condicionante de la propia situación de vulnerabilidad, pero aquí surgen algunas diferencias: se le menciona en términos individuales, considerando que los niños y adolescentes deberían superar las condiciones de vida de sus familias o bien, se les considera como parte del problema sobre el cual intervenir o finalmente, se estipula que uno de los objetivos es promover la vinculación con estas familias.

Resulta interesante observar como de este modo se establecen para los programas, las problemáticas sociales sobre las cuales intervenir. La familia puede ser un problema o puede ser parte del problema y de aquí surgen diferentes "modos de hacer" de la política social. Así, se cuelan discursos que toman la problemática de la pobreza en términos individuales de superación de la misma, al tiempo que se vislumbran algunos discursos que por el contrario, consideran a las familias también afectadas por las condiciones de pobreza, las cuales no podrían garantizar los derechos de niños, niñas y adolescentes. Esto aparece mencionado específicamente para el caso de la provincia de Buenos Aires.

\section{ALGUNAS CONSIDERACIONES FINALES}

Se ha intentado presentar un análisis de las modalidades en que se expresan las tensiones derivadas de dos tendencias coexistentes $y$ divergentes en políticas y programas concretos, tales como: el paradigma de ampliación de ciudadanía y derechos de la infancia y la adolescencia, así como, la despolitización y reenmarcamiento de la cuestión social en términos de pobreza y exclusión, y no en virtud de las condiciones de producción de la desigualdad.

El marco de exclusión social, tal como ha sido analizado por autores como por ejemplo Saraví (2006), para el caso de jóvenes argentinos y mexicanos, es de utilidad considerar cómo operan los procesos altamente individualizados de integración y exclusión en modelar trayectorias vitales. Por otro lado, investigaciones en los contextos británico y canadiense han mostrado cómo la inclusión del discurso de la exclusión social en el centro del denominado "estado de inversión social" (Giddens, 1998), ha desplazado el problema de la desigualdad del campo de acción y preocupación estatal. Al mismo tiempo, ha servido para reinscribir el discurso individualizante de la pobreza en el corazón de la ciudadanía social, limitando y restringiendo el alcance de esta última (Lister, 2007 y 2002; Mayer, 2008; Jenson, 2009).

La inclusión del enfoque de derechos en las políticas sociales para la infancia ha propiciado, en este contexto, una movilización de actores que visibiliza los problemas de este sector y obliga a los actores nacionales e internacionales, que de otro modo probablemente no se verían compelidos, a proponer un lugar destacado en la agenda institucional para los problemas de la infancia y adolescencia.

No obstante, al analizar los procesos de institucionalización e interpretación mediante los cuales estas ideas son incorporadas en el diseño de los programas y políticas sociales, lo que hallamos es su re-enmarcamiento en definiciones de problemas y estrategias altamente individualizados y psicologizados, cuyo efecto dificultosamente pueda encontrarse al nivel de la transformación de las dinámicas que conducen a la exclusión y restricción de derechos de las generaciones más jóvenes. A ello se suma la prevalencia de modalidades de diseño que facilitan a los funcionarios el pensar en una escala programática y no política, generando acciones cuyo mismo recorte poblacional, asignación de recursos y dinámica de funcionamiento 
restringen lo que aparece a la hora de definir objetivos $y$ problemas.

Finalmente, la tensión inherente a las políticas sociales para la infancia y la adolescencia, derivada de su doble función de incorporación de las nuevas generaciones y de reproducción de la sociedad (Llobet, 2009), en un contexto de securitización de la cuestión social (Kessler, 2004), permite que estas tensiones se inscriban en una semántica de restricción de la inclusión (Litichever, 2009) y de privilegio de los resultados de control social. De este modo, las y los beneficiarios de los programas sociales son construidos como tales, a condición de que acepten las reglas de definición de sus identidades por parte de los programas. Lo que queda por fuera es, ciertamente, una discusión respecto a cómo esta transformación de los sujetos en "beneficiarios" los acercaría a logros en términos de ciudadanía social e inclusión en la sociedad.

No obstante, los debates políticos, los actores presentes $y$ las negociaciones situadas permiten señalar que aparecen dos tendencias parcialmente diferentes en la ciudad de Buenos Aires y en la Provincia de Buenos Aires. La primera mantiene una lógica de mayor focalización a la vez, aparentemente menor diálogo y articulación entre las diferentes áreas, un fuerte sesgo moralista, con raíces en la consideración de los individuos como responsables de su inclusión (y consecuentemente culpables de su pobreza) y una retórica de derechos. En la Provincia de Buenos Aires, comienzan a aparecer rasgos de mayor intersectorialdad, cierta inclusión de las comunidades y las familias como aspectos que hacen a la atención de la vulnerabilidad social, con tensiones entre un enfoque comunitarista que ubica actores barriales y un enfoque moralista respecto de "las familias" consideradas como actores homogéneos y normativamente estructurados para facilitar la inclusión social.

Es necesario continuar profundizando los alcances y manifestaciones de estas diferencias, que en tanto el contexto sociocultural de posibilidad para la implementación del paradigma de derechos de la infancia, dan cuenta también de sus límites actuales.

\section{BIBLIOGRAFÍA}

LIBROS

Baratta, Alessandro. "Infancia y democracia". Infancia, ley y democracia en América Latina. García Méndez, Emilio y Beloff, Mary (comps.). Santa Fé de Bogotá/ Buenos Aires: Edit. Temis/Depalma, 1999 .

Bourdieu, Pierre y Wacquant, Loic. Una invitación a la sociología reflexiva. Buenos Aires: Siglo XXI, 2005.

Fleury Teixeira, Sonia. Estados sin ciudadanos: seguridad social en América Latina. Buenos Aires: Lugar Editorial, 1997.

Foucault, Michel. The history of sexuality. Vol. 1. New York: Pantheon Books, 1978.

Fraser, Nancy. Iustitia interrupta. Reflexiones críticas desde la posición "postsocialista". Santa Fé de Bogotá: Siglo del Hombre Editores, 1997.

García Méndez, Emilio. "La Convención Internacional de los Derechos del Niño: del menor como objeto de la compasión represión a la infancia adolescencia como sujetos de derechos". Derecho de la infancialadolescencia en América latina: de la situación irregular a la protección integral. Ibagué, Venezuela: Forum Pacis, 1997.

Giddens, Anthony. The third way: the renewal of social democracy. Cambridge: Polity Press, 1998.

Guemureman, Silvia y Daroqui, Alcira. La niñez ajusticiada. Buenos Aires, Argentina: Editorial Del Puerto, 2001.

Haney, Lynne. Inventing the needy: gender and the politics of welfare in Hungary. Berkeley: U. California Press, 2002.

Jorgensen, S. "Freedom from want and freedom from fear: new frontiers in social policy". Social protection initiatives for children, women and families. Minujin, A. y Delamonica, E. (eds.). The New School: UNICEF, 2007.

Kessler, Gabriel. Sociología del delito amateur. Buenos Aires: Paidós, 2004.

Llobet, Valeria. “¿Retratos de niño? Políticas sociales y derechos de niñ@s en situación 
de calle". La cuestión de la infancia. Entre la escuela, la calle y el shopping. Carli, Sandra (comp.). Buenos Aires: Paidós editorial, 2006.

Llobet, Valeria. ¿Fábricas de niños? Las instituciones en la era de los derechos. Buenos Aires: Editorial Novedades Educativas, 2009.

Llobet, Valeria. "Género y políticas sociales para la infancia, ¿una relación ausente?". Politica social en América Latina y género: configuraciones/ reconfiguraciones en la participación de las mujeres. Ortale, S. y Enriquez Rosas, M. (comps.). Ed. Biblos, 2009a (en prensa).

Llobet, Valeria. "Las políticas sociales para la infancia $y$ la adolescencia en Argentina $y$ el paradigma internacional de derechos humanos". Politicas Sociales Latinoamericanas. Perspectivas comparadas. México: Universidad Autónoma del Estado de México y Editorial Porrúa, 2009b (en prensa).

Llobet, Valeria y Litichever, Cecilia. "Desigualdad e inclusión social ¿Qué proponen los programas de atención a niños, niñas y adolescentes?". Jóvenes y desigualdad. México: Editorial Porrúa y Universidad Autónoma del Estado de México, 2010.

Merklen, Denis. Pobres ciudadanos. Las clases populares en la era democrática (Argentina, 1983-2003). Buenos Aires: Gorla, 2005.

Stake, Robert. Investigación con estudio de casos. Madrid: Morata, 1998.

Yin, Robert. Applications of case study research. Beverly Hills, CA: Sage Publishing, 1993.

REVISTAS

Barrientos, Armando y Santibáñez, Claudio. "New forms of social assistance and the evolution of social protection in Latin America". Cambridge Journals. 2009.

Belmartino, Susana; Levin, S. y Repetto, F. "Políticas sociales $y$ derechos sociales en la Argentina: breve historia de un retroceso". Socialis 5. Argentina, 2001.

Cillero Bruñol, Miguel. "De la proclamación a la protección efectiva". Revista Justicia y Derechos del Niño 3. UNICEF, 2001.

Dobrowlsky, Alexandra y Lister, Ruth. "Social exclusion and changes to citizenship: women and children, minorities and migrants in Britain". Paper prepared for Canadian Political Science Association Annual Meetings University of Western. London Ontario, junio 2005.

Elizabeth, Vivienne y Larner, Wendy. "Racializing the "social development" state: investing in children in Aotearoa/ New Zealand". Social Politics: International Studies in Gender, State and Society 16(1). 2009: 132-158.

Fernando, Jude L. "Children's rights: beyond the impasse". Annals of the American Academy of Political and Social Science 575. 2001: 8-24.

Fonseca, C. y Cardarello, A. "Derechos de los más y menos humanos". Derechos Humanos, tribunales y policías en Argentina y Brasil. Tiscornia, S. y Pita, M. V. (comps.). Buenos Aires, Antropofagia, 2005.

Fraser, Nancy. "La lucha por las necesidades: esbozo de una teoría crítica socialista feminista de la cultura política del capitalismo tardío". Debate Feminista. 1991.

Gentile, María Florencia. "La interacción cotidiana en un centro para niños y adolescentes en situación de calle, desde la experiencia de los chicos que participan". Ponencia $1^{\circ}$ Reunión Nacional de Investigadores/as en Juventudes (RENIJ). Universidad Nacional de La Plata, 2007.

Jenson, Jane. "Lost in translation: the social investment perspective and gender equality". Social Politics. International Studies in Gender, State and Society 16 (4). 2009: 446-483.

Lister, Ruth. "Investing in the citizen-workers of the future: new labour's 'Third Way' in welfare reform". Ponencia en la reunión anual de la Asociación Americana de 
Ciencias Políticas. Boston, Agosto de 2002. En: <http://www.fas.umontreal.ca/ $\mathrm{POL} /$ cohesionsociale $>$ [consultado el 3 de julio de 2007].

Lister, Ruth. "Children (but not women) first: new Labour, child welfare and gender". Critical Social Policy 26 (2). 2006: 315335.

Lister, Ruth. Social citizenship in new labour's new "active" welfare state. Mimeo. 2007.

Llobet, Valeria. "Las políticas sociales para la infancia, la psicología y el problema del reconocimiento". Revista Investigación en Psicología 14 (II). 2009b.

Padamsee, Tasleem. "Culture in connection: re-contextualizing ideational processes in the analysis of policy development". Social Politics: International Studies in Gender, State and Society 16 (4). 2009: 413-445.

Saraví, Gonzalo. "Biografías de exclusión: desventajas y juventud en Argentina". Perfiles Latinoamericanos 28. México. Facultad Latinoamericana de Ciencias Sociales, 2006: 83-116.

Tellis, Winston. "Application of a case study methodology". The Qualitative Report 3 (3). 1997. En: <http://www.nova.edu/ssss/ QR/QR3-3/tellis2.html>

Therborn, Göran. "Child politics: dimensions and perspectives". Childhood 3. 1996: $29-44$.

Unidad de Información, Monitoreo y Evaluación (UIMYe). "La situación social de los jóvenes beneficiarios del Programa Ciudadanía Porteña. La situación en el 2009". Serie Informes de Condiciones de Vida. Ministerio de Desarrollo Social, 2010.

UNRISD. Targeting and universalism in poverty reduction. Geneva: UNRISD, 2005.

Villalta, Carla. "La administración de la infancia en debate. Entre tensiones y reconfiguraciones institucionales". Estudios en Antropología Social 1 (2). Centro de Antropología Social, Instituto de Desarrollo Económico y Social, 2010.
Watson, Sophie. "Foucault and the study of social policy". Rethinking Social Policy. Lewis, Gail; Gewirtz, Sharon y Clarke, John (comp.). London: Sage Open University, 2000.

TESIS

Litichever, Cecilia. "Trayectoria institucional y ciudadanía de chicos y chicas en situación de calle". [Tesis de Maestría en Diseño y Gestión de Programas y Políticas Sociales]. Flacso, 2009. En: <http://flacsoandes.org/web/cms. php?c $=43>$

Llobet, Valeria. "La convención de derechos de niños, la ciudadanía y los chicos de la calle". [Tesis de doctorado no publicada]. Argentina: Universidad de Buenos Aires, Facultad de Psicología, 2009.

PÁGINAS WEB

Ministerio de Desarrollo Social (MDS). La bisagra. 2007 . En: <www. desarrollosocial.gov.ar>

Ministerio de Desarrollo Social de la Ciudad Autónoma de Buenos Aires. En: http:// www.buenosaires.gov.ar/areas/des social/niniez_adolescencia/?menu_ $\mathrm{id}=167$

Programa de las Naciones Unidas para el Desarrollo (PNUD). Informe sobre Desarrollo Humano. Derechos Humanos y Desarrollo Humano, 2000. En <http:// hdr.undp.org/es/informes/mundial/ idh2010/>

Instituto Nacional de Estadísticas y Censos (INDEC). Encuesta Permanente de Hogares (EPH). Segundo semestre de 2006. En: <http://www.indec.gov.ar>

UNICEF. Datos sobre infancia en la Argentina. En: <http://www.unicef.org/argentina/ spanish/overview_11124.htm>

Fecha de ingreso: 12/12/2011 Fecha de aprobación: 08/03/2012 